\title{
Better vascular healing of ultrathin strut biodegradable-polymer sirolimus-eluting stents in patients with acute coronary syndrome
}

\section{Akito Kawamura}

Osaka Rosai Hospital: Osaka Rosai Byoin

\section{Yasuyuki Egami}

Osaka Rosai Hospital: Osaka Rosai Byoin

Shodai Kawanami

Osaka Rosai Hospital: Osaka Rosai Byoin

Hiroki Sugae

Osaka Rosai Hospital: Osaka Rosai Byoin

Kohei Ukita

Osaka Rosai Hospital: Osaka Rosai Byoin

Hitoshi Nakamura

Osaka Rosai Hospital: Osaka Rosai Byoin

Yutaka Matsuhiro

Osaka Rosai Hospital: Osaka Rosai Byoin

Koji Yasumoto

Osaka Rosai Hospital: Osaka Rosai Byoin

Masaki Tsuda

Osaka Rosai Hospital: Osaka Rosai Byoin

Naotaka Okamoto

Osaka Rosai Hospital: Osaka Rosai Byoin

Yasuharu Matsunaga-Lee

Osaka Rosai Hospital: Osaka Rosai Byoin

Masamichi Yano

Osaka Rosai Hospital: Osaka Rosai Byoin

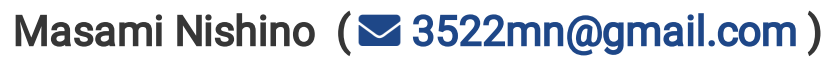

Osaka Rosai Hospital https://orcid.org/0000-0003-0309-7023

Jun Tanouchi

Osaka Rosai Hospital: Osaka Rosai Byoin 
Keywords: optical coherence tomography, vascular healing, ultrathin strut, ACS

Posted Date: August 11th, 2021

DOI: https://doi.org/10.21203/rs.3.rs-791784/v1

License: (c) (1) This work is licensed under a Creative Commons Attribution 4.0 International License. Read Full License 


\section{Abstract}

This study aimed to compare the strut coverage between Orsiro ultrathin struts biodegradable polymer sirolimus-eluting stents (O-SES) and Xience thin struts durable polymer everolimus-eluting stents (X-EES) in acute coronary syndrome (ACS) patients using optical coherence tomography (OCT). In BIOSTEMI trial, O-SESs were superior to X-EESs with respect to target lesion failure (TLF) in ACS patients. However, there were few reports comparing intravascular imaging between the two stents in ACS. Between August 2016 and February 2020, 50 lesions from 50 ACS patients who underwent OCT-guided percutaneous coronary intervention $(\mathrm{PCl})$ were enrolled. We compared mid-term vascular healing using OCT between O-SESs and X-EESs at 8-month after stenting. The protocol was approved by the Osaka Rosai Hospiral ethics committee. Among 50 lesions, the X-EES group consisted of 25 lesions and the O-SES of 25 lesions. The percentage of covered strut, the percentage of malapposed strut and mean neointimal thickness at 8month were evaluated. In the 8-month OCT analysis, the proportion of covered strut was significantly higher in the O-SES group than in in the X-EES group ( $97.3 \%$ vs. $86.0 \% ; p=0.001)$. On the other hand, there were no significant differences in the frequency of malapposed strut $(0.4 \%$ vs $1.0 \%, p=0.238)$. The O-SES group had the tendency of thinner neointima compared to the X-EES group $(60 \mu \mathrm{m}$ vs $76 \mu \mathrm{m}, \mathrm{p}=$ 0.089). Compared to X-EESs, O-SESs showed better mid-term vascular healing and tended to have thinner neointima in ACS patients. Ultra-thin strut may play a key role in better vascular healing.

\section{Introduction}

Primary percutaneous coronary intervention $(\mathrm{PCl})$ is the preferred strategy to restore myocardial perfusion in acute coronary syndrome (ACS) patients.[1] In addition, drug eluting stents (DESs) have improved the clinical outcome in ACS patients as compared to bare metal stents (BMSs).[2] New generation DESs have had a better long-term outcome than 1st -generation DESs.[3] In the BIOSTEMI trials, the Orsiro ultrathin strut biodegradable polymer sirolimus-eluting stents (O-SESs) were superior to the Xience thin strut durable polymer everolimus-eluting stents (X-EESs) in terms of the target lesion failure at 1 year and the difference was driven mainly by a reduced ischemia-driven target lesion revascularization (TLR) in patients treated by the 0-SESs.[4] The superiority was maintained at the 2-year follow-up and the incidence of TLR was significantly lower for O-SESs than X-EESs.[5] However, the reason for the outcome in the recent study was poorly understood. There have been few reports showing the pathophysiological insights such as intravascular imaging studies and histopathological analyses of the differences between X-EESs and O-SESs in ACS patients. OCT imaging delivers a higher resolution and can precisely detect the covered strut and neointimal hyperplasia. $[6,7]$ In the present study, we compared the OCT parameters such as the percentage of covered struts, percentage of malapposed struts, and mean neointimal thickness at 8-months between O-SESs and X-EESs to elucidate the mechanism of the previous study.

\section{Methods}




\section{Study population}

Consecutive ACS patients, who underwent an OCT-guided PCl, using O-SESs or X-EESs and that underwent an 8-month follow-up OCT in our hospital between August 2016 and February 2020, were enrolled in the study. The ACS patients included those with unstable angina pectoris (UAP), non-STelevation myocardial infractions (NSTEMIs) and STEMIs. The ACS patients who were treated with DESs (O-SESs or X-EESs) and had a clear OCT image at the 8-month follow-up were included. The choice of those 2 stents depended on each operator's discretion. The study exclusion criteria were in-stent restenosis, hemodynamic instability, an age less than 18 years, and a life expectancy of less than six months due to a non-cardiac condition. Written informed consent was obtained from all participating patients and the protocol was approved by the Osaka Rosai Hospiral ethics committee. This study conformed to the ethical guidelines outlined in the Declaration of Helsinki.

\section{Stent type}

The O-SESs (Orsiro; Biotronik; Bulach, Switzerland) consisted of an ultrathin strut $(60 \mu \mathrm{m}$ for stent diameters $\leqq 3.0 \mathrm{~mm}$ and $80 \mu \mathrm{m}$ for stent diameters $>3.0 \mathrm{~mm}$ ) cobalt-chromium metallic stent platform covered by an amorphous, hydrogen-rich, silicon-carbide passive layer and an asymmetric biodegradable poly-L-lactic acid polymer active coating that released sirolimus at a dose of $1.4 \mu \mathrm{g}$ per mm2 of the stent surface, which degraded over a period of 12 to 24 months.[8] On the other hand, the X-EESs (Xience Alpine/Sierra; Abbott Vascular, Abbott Park, IL, USA) consisted of a thin strut $(81 \mu \mathrm{m})$ cobalt-chromium stent platform that released everolimus from a durable polymer.

\section{Procedural and medical management}

$\mathrm{PCl}$ for culprit lesions was performed according to the standard techniques. Thrombus aspiration, excimer laser coronary angioplasty (ELCA), direct stenting and post-dilation were left to the operator's discretion, and the operator referred to the OCT findings to determine the strategy. Before the intervention, all patients were pretreated with $200 \mathrm{mg}$ of aspirin and a loading dose of P2Y12inhibitor (300mg of clopidogrel or $20 \mathrm{mg}$ of prasugrel). After the intervention, all patients received a dual antiplatelet therapy (DAPT: aspirin $100 \mathrm{mg}$ and a P2Y12R inhibitor [75mg of clopidogrel or $3.75 \mathrm{mg}$ of prasugrel]) daily for at least 6 months.

\section{OCT image acquisition}

The OCT system used in this study consisted of a computer, monitor display, and interface unit (C7 OCT System, Abbott Vascular, Santa Clare, CA, USA). The patients received heparin intravenously before the OCT procedure. Using the C7 OCT system, a conventional angioplasty guidewire (0.014-inch) was advanced distal to the region of interest, then the OCT catheter (Dragonfly, Abbott Vascular, Santa Clara, CA, USA) was advanced over the guidewire beyond the region of interest. During the imaging acquisition, blood was displaced by an injection of contrast media. In general, in the patients presenting with Thrombolysis In Myocardial Infarction (TIMI) flow grades of 2 and 3, the OCT was performed before any intervention, while for cases with a TIMI of 0 or 1 , the OCT was performed after a thrombectomy or pre- 
dilatation using only small sized balloons ( $\leq 2.0 \mathrm{~mm}$ balloon). $[9,10]$ The images were calibrated by an automated adjustment of the Z-offset and the automated pullback was set at 18 or $36 \mathrm{~mm} / \mathrm{s}$. Data were acquired using a commercially available OCT system (C7 OCT System and Dragonfly imaging catheter, Abbott Vascular, Santa Clare, CA, USA) and were digitally stored.

\section{OCT image analysis}

We performed quantitative and qualitative OCT analyses using dedicated software (Off-line Review Software, version E.0.2, Abbott Vascular, Santa Clara, CA, USA). In the pre-procedural, post procedural, and 8-month follow-up OCT data, all cross-sectional images were initially screened for a quality assessment and excluded from analysis if any portion of the stent was out of the screen or if the image quality was considered poor due to residual blood, artifact, or reverberation. Bifurcations with major side branches, which were defined as side branches $>45^{\circ}$, and stent overlapping segments were also excluded.[11] Quantitative (i.e., luminal areas and diameters) and qualitative measurements were performed on every $1 \mathrm{~mm}$ frame along the entire target segment.

In the pre-procedural OCT data, the assessment of the lesion morphology was performed at the culprit site and the lesions were categorized according to their most prevalent component as follows: (a) plaque rupture and (b) intact fibrous cap. Plaque rupture was defined as the presence of a fibrous cap discontinuity leading to a communication between the inner core of the plaque and the lumen. An intact fibrous cap included both definite (the presence of an attached thrombus overlying an intact and visualized plaque) and probable erosions, defined as a luminal irregularity without a thrombus or thrombus without a superficial lipid or calcified plaque in the proximity of the thrombus. In addition, intact fibrous caps included smooth plaque without evidence of a rupture or thrombus.[12] Thrombus at the culprit site was divided into red thrombus or white thrombus. Red thrombus appeared as a mass with a high backscattering and high attenuation and white thrombus as a homogeneous mass with less backscattering and a low attenuation.[13] We recorded the maximum thrombus area and length of the thrombus within the culprit lesion. The minimum lumen area (MLA) was derived from an automatic lumen segmentation within the region of interest.

In the post-procedural OCT data, the minimum lumen area (MLA) was derived from an automatic lumen segmentation within the stented lesion. In addition, we evaluated the minimum stent area (MSA) at the MLA site and manually traced the stent area by interpolated contours connecting the center point of the luminal surface of each detected metallic strut. The proximal and distal reference areas were measured at the largest lumen within $5 \mathrm{~mm}$ of the proximal and distal edges. The strut-lumen distance was determined based on automated measurements performed from the center of the strut blooming to the luminal contour of the artery wall. A malapposed strut was defined as having a strut-lumen distance (XEES $>89 \mu \mathrm{m}, \mathrm{O}-\mathrm{SES}>67 \mu \mathrm{m}$, and $>87 \mu \mathrm{m}$ for the $2.25,2.5$, and 3.0 stent diameters and 3.5 and 4.0 stent diameters, respectively). A stent edge dissection was defined as the disruption of the vessel luminal surface with a visible flap at the stent edge or within $5 \mathrm{~mm}$ of the proximal and distal reference segments. The in-stent tissue protrusions were divided into 3 categories: smooth protrusions, disrupted fibrous tissue protrusions, and irregular protrusions.[14] Smooth protrusions were defined as the bowing of the 
plaque into the lumen between the stent struts, without any intimal disruption, appearing as a smooth semicircular arc connecting the adjacent struts, and likely representing a compression of the soft plaque by the stent. Disrupted fibrous tissue protrusions were defined as a disruption of the underlying fibrous tissue protruding from the stent struts into the lumen. Irregular protrusions were defined as protrusions of the material with an irregular surface into the lumen between the stent struts. As the struts are occasionally buried within the intima, we included only in-stent protrusions with a maximal height of $\geq$ $100 \mu \mathrm{m}$ for the analysis in the current study.[14]

In the 8-month follow-up OCT data, the neointimal hyperplasia $(\mathrm{NIH})$ thickness was defined as the distance between the endoluminal surface of the strut reflection and the lumen contour. The mean $\mathrm{NIH}$ thickness (total $\mathrm{NIH}$ thickness divided by the number of total struts), maximum NIH thickness, and minimum NIH thickness was calculated for each lesion.[15] Struts were categorized as malapposed struts, uncovered struts, and covered struts. Malapposed struts were defined in the same way as the postprocedural OCT analysis. Struts were classified as uncovered if any part of the strut was visibly exposed to the lumen. In addition, uncovered struts were categorized as apposed or malapposed struts.[16] The representative images of covered, uncovered and malapposed struts were shown in Fig. 1. The percentage of uncovered struts was calculated as the number of uncovered struts divided by the total number of analyzed struts for each lesion. We compared the OCT parameters including the percentage of uncovered struts, malapposed struts, and the mean NIH thickness between the X-EES and the O-SES groups.

Two independent readers in our institution, who were blinded to the patient information, retrospectively performed quantitative and qualitative OCT analyses. If there was an ambiguous OCT imaging of the coverage, this was argued between the two observers and a final determination was made. To measure inter-observer reproducibility, the percentage of uncovered struts and the percentage of malapposed struts were compared using the Bland-Altman method in 10 randomly selected OCT image.[17]

\section{Statistical analysis}

All statistical analyses were performed using JMP version 14 software (SAS Institute, Inc., Cary, North Carolina, USA) and the statistical significance was assessed at a p-level of 0.05 . The continuous variables are expressed as the mean \pm SD or median (interquartile range) and categorical variables as the count (percentage). For the continuous variables, the difference between the two groups were made with the nonparametric Mann-Whitney $U$ test, and the categorical variables were compared with a Fisher exact test at the lesion level. All variables were analyzed at the lesion level. The pre-procedural, post-procedural, and 8-month follow-up OCT parameters were compared in all lesions.

\section{Results}

\section{Baseline and procedural characteristics}


There were 25 lesions with X-EESs and 25 with O-SESs for the serial OCT-analysis. The baseline patient clinical characteristics and medications are summarized in Table 1. There were no significant differences in the age, sex, or known coronary risk factors between the two groups. Antiplatelet therapy with single or double agents were continued for all patients throughout follow-up. In addition, the use of statins, angiotensin converting enzyme inhibitor/angiotensin receptor blockers and beta-blockers at baseline and the 8-month follow-up OCT were comparable between the two groups. In terms of stent-related complications, there were no cases of stent thromboses or target lesion revascularizations in either group during the 8-month follow-up period. 
Table 1

Baseline Patient Characteristics

\begin{tabular}{|c|c|c|c|}
\hline & $X$-EES (n= 25) & O-SES $(n=25)$ & $P$ value \\
\hline Age, years & $68.0(59.0-73.5)$ & $65.0(58.5-71.5)$ & 0.491 \\
\hline Male sex & $19(76.0)$ & $24(96.0)$ & 0.098 \\
\hline \multicolumn{4}{|l|}{ Coronary risk factors } \\
\hline Diabetes mellitus & $5(20.0)$ & $11(44.0)$ & 0.128 \\
\hline Hypertension & $18(72.0)$ & $19(76.0)$ & 1.000 \\
\hline Dyslipidemia & $13(52.0)$ & $19(76.0)$ & 0.140 \\
\hline Hemodialysis & $0(0)$ & $0(0)$ & 1.000 \\
\hline Current smoker & $17(68.0)$ & $20(80.0)$ & 0.520 \\
\hline Prior myocardial infraction & $1(4.0)$ & $2(8.0)$ & 1.000 \\
\hline $\mathrm{BMI}, \mathrm{kg} / \mathrm{m} 2$ & $24.3(20.9-25.6)$ & $25.2(22.6-27.7)$ & 0.197 \\
\hline \multicolumn{4}{|l|}{ Medications at baseline } \\
\hline Antiplatelet therapy & $2(8.0)$ & $6(24.0)$ & 0.247 \\
\hline Oral anticoaglant agent & $1(4.0)$ & $1(4.0)$ & 1.000 \\
\hline Statin & $2(8.0)$ & $8(32.0)$ & 0.074 \\
\hline ACE-I or ARB & $7(28.0)$ & $8(32.0)$ & 1.000 \\
\hline$\beta$-blocker & $0(0)$ & $3(12.0)$ & 0.235 \\
\hline \multicolumn{4}{|l|}{ Medications at follow-up } \\
\hline Antiplatelet therapy & $25(100)$ & $25(100)$ & 1.000 \\
\hline Oral anticoaglant agent & $2(8.0)$ & $2(8.0)$ & 1.000 \\
\hline Statin & $24(96.0)$ & $25(100)$ & 1.000 \\
\hline ACE-I or ARB & $23(92.0)$ & $22(88.0)$ & 1.000 \\
\hline$\beta$-blocker & $18(72.0)$ & $18(72.0)$ & 1.000 \\
\hline
\end{tabular}

The procedural characteristics are summarized in Table 2. The lesion locations, stent diameter and stent length were comparable between the groups. The usage rate of a thrombus aspiration catheter and ELCA was similar between the groups. 
Table 2

Procedural Characteristics

\section{$X$-EES $(n=25)$ \\ O-SES $(n=25) \quad$ P value}

The types of ACS

0.525

UAP

$2(8.0)$

$5(20.0)$

NSTEMI

7 (28.0)

$6(24.0)$

STEMI

$16(64.0)$

$14(56.0)$

Lesion location

0.142

Right coronary artery

$12(48.0)$

$7(28.0)$

Left anterior descending

$9(36.0)$

$16(64.0)$

Left circumflex

4 (16.0)

2 (8.0)

Thrombus aspirations

$16(64.0)$

$12(48.0)$

0.393

ELCA

$9(36.0)$

$6(24.0)$

0.538

Direct stenting

7 (28.0)

$8(32.0)$

1.000

Post dilation

14 (56.0)

8 (32.0)

0.154

Stent size, $\mathrm{mm}$

$3.25(2.75-3.38)$

3.00 (3.00-3.50)

0.333

Stent length, $\mathrm{mm}$

$23(18-28)$

$18(15-26)$

0.148

TIMI flow after PCI 0, 1, 2, 3

$0,0,1,24$

$0,1,3,21$

0.349

ACS $=$ acute coronary syndrome, UAP = unstable angina pectoris, NSTEMI = non-ST-elevation myocardial infraction, STEMI = ST-elevation myocardial infraction, ELCA = excimer laser coronary angioplasty, TIMI = Thrombolysis in Myocardial Infraction, $\mathrm{PCl}$ = percutaneous coronary intervention. The other abbreviations are the same as in Table 1.

\section{OCT findings}

There were no OCT procedural-associated complications in this study. The results of the pre-procedural and post-procedural OCT findings are summarized in Table 3. There were no significant differences in the pre-procedural and post-procedural OCT parameters between the two groups. The results of the 8-month OCT follow-up analysis are summarized in Table 4. The median follow-up periods were similar between the two groups. In the 8-month OCT follow-up analysis, the mean $\mathrm{NIH}$ thickness tended to be thinner in the O-SES group than X-EES group $(60 \mu \mathrm{m}$ vs. $76 \mu \mathrm{m}, \mathrm{P}=0.089)$. The percentage of covered struts in the stent-lever analysis was higher in the O-SES group than $\mathrm{X}$-EES group $(97.3 \% \mathrm{vs.} 86.0 \%, \mathrm{P}=0.001)$. The proportion of malapposed struts was similar between the 2 groups $(0.4 \%$ vs. $1.0 \%, P=0.238)$. The other parameters including the maximum NIH thickness, minimum lumen area, and minimum stent area did not differ between the groups. 
Table 3

OCT Findings at Pre-Procedural and Post-Procedural PCI

\section{$X$-EES $(n=25) \quad$ O-SES $(n=25) \quad P$ value}

Pre-procedural OCT parameters

Minimum lumen area, $\mathrm{mm} 2$

$1.08(0.94-$

1.38)

$1.21(0.91-$

0.384

White thrombus, $\mathrm{n}$

$22(91.7)$

$18(72.0)$

0.138

Red thrombus, $\mathrm{n}$

$9(37.5)$

$14(56.0)$

0.256

Maximum thrombus area, $\mathrm{mm} 2$

$0.73(0.25-$

$0.83(0.05-$

0.439

$1.55)$

1.37)

Thrombus length, $\mathrm{mm}$

$4.7(2.0-6.6)$

$3.4(1.0-5.5)$

0.164

Ruptured plaque, $n$

18 (75.0)

$12(54.6)$

0.217

Intact fibrous cap, $\mathrm{n}$

$6(25.0)$

$10(45.5)$

0.217

Post-procedural OCT parameters

Minimum lumen area, $\mathrm{mm} 2$

$5.54(4.32-$

6.58)

$5.63(3.91-$

0.614

Minimum stent area, $\mathrm{mm} 2$

$\begin{array}{ll}6.06(5.00-7.32) & 6.20(4.98- \\ & 7.64)\end{array}$

0.541

Maximum malapposition struts lumen distance,

$240(165-445)$

$260(125-320)$

0.554 $\mu \mathrm{m}$

Maximum malapposition struts length, $\mathrm{mm}$

$1.6(0.8-3.3)$

$1.5(0.6-2.3)$

0.240

Main protrusion characteristics

0.349

Irregular proturusion, $\mathrm{n}$

$23(92.0)$

$21(84.0)$

Smoothe protrusion, $\mathrm{n}$

$1(4.0)$

$4(16.0)$

Disrupted protrusion, $\mathrm{n}$

$1(4.0)$

$0(0)$

Maximum protruding area, $\mathrm{mm} 2$

$0.52(0.25-$

$0.94)$

$0.32(0.15-$

0.084

Maximum protruding length, $\mathrm{mm}$

$2.6(1.9-6.1)$

$2.2(1.2-4.9)$

0.244

Edge dissection, $\mathrm{n}$

$0(0)$

$1(4.0)$

1.000

$\mathrm{OCT}=$ optical coherence tomography, $\mathrm{PCl}=$ percutaneous coronary intervention. The other abbreviations are the same as in Table 1. 
Table 4

OCT Findings at 8-month Follow-up

\begin{tabular}{|llll|}
\hline & X-EES $(\mathbf{n}=\mathbf{2 5})$ & O-SES $(\mathbf{n}=\mathbf{2 5})$ & P value \\
\hline Mean follow-up, days & $253(246-289)$ & $239(223-266)$ & 0.073 \\
\hline Stent level analysis & & & \\
\hline Stent struts & $115(87-194)$ & $159(122-213)$ & 0.073 \\
\hline Percentage of covered struts & $86.0(63.0-96.1)$ & $97.3(94.2-99.3)$ & 0.001 \\
\hline Percentage of malapposed struts & $1.0(0-6.0)$ & $0.4(0-1.9)$ & 0.238 \\
\hline Minimum NIH thickness, $\mu \mathrm{m}$ & $10(10-10)$ & $10(10-10)$ & 0.641 \\
\hline Maximum NIH thickness, $\mu \mathrm{m}$ & $250(200-320)$ & $230(125-290)$ & 0.120 \\
\hline Mean NIH thickness, $\mu \mathrm{m}$ & $76(52-99)$ & $60(35-80)$ & 0.089 \\
\hline Minimum lumen area, mm2 & $4.64(3.91-5.53)$ & $5.40(4.14-7.08)$ & 0.181 \\
\hline Minimum stent area, mm2 & $5.58(4.56-6.88)$ & $5.93(5.07-7.87)$ & 0.443 \\
\hline NIH = neointimal hyperplasia. The other abbreviations are the same as in Table 1 and Table 3. \\
\hline
\end{tabular}

\section{Discussion}

The main findings in this study were that in the ACS patients, (1) the proportion of uncovered struts was significantly lower in the O-SES group than X-EES group and (2) the mean NIH thickness tended to be thinner in the O-SES group than X-EES group. We demonstrated the mid-term vascular healing in terms of an OCT analysis between the O-SESs and X-EESs in ACS patients.

Several randomized clinical trials have shown that DESs dramatically reduce the incidence of angiographic restenosis and incidence of repeat revascularizations compared to bare metal stents.[18] After a technical improvement, the new-generation DESs have emerged, which combine thin strut stent platforms with biocompatible durable polymer coatings. However, in the era of new-generation DESs, stent thrombosis and in-stent restenosis are the major concerns of the contemporary PCI.[19, 20] 0-SESs consist of ultra-thin strut platforms and biodegradable polymer coatings, which have been designed to minimize inflammation and vascular injury and to promote rapid endothelialization and a thinner neointima.[21, 22] Therefore, the frequency of stent thromboses and in-stent restenosis were expected to decrease in patients undergoing a PCI with O-SESs. Moreover, in the setting of ACS, the plaque of the culprit lesion is more vulnerable and inflammatory reactions are high.[23] In such a situation, the advantage of O-SESs can be stronger and indicate a better clinical outcome in terms of stent thromboses and in-stent restenosis. In fact, the BIOSTEMI trial first showed that O-SESs had a lower incidence of clinical indicated TLR in patients with STEMIs than X-EESs.[4] In the BIOFLOW $\otimes$ trial, definite or probable late or very late stent thromboses were significantly lower with O-SESs than X-EESs. [24] However, the 
pathophysiological concept for a reduction in the clinical events in patients treated with O-SESs as compared to X-EESs has not been fully understood and has warranted further study such as intravascular imaging studies and histopathological analyses. The current study using intravascular imaging, to the best of our knowledge, has for the first time demonstrated that O-SESs showed a better mid-term vascular healing and thinner neointimal hyperplasia than X-EESs among ACS patients. With respect to vascular healing, a similar outcome was reported in the HATTRICK OCT trial, but the comparator was not X-EESs but was durable polymer zotarolimus-eluting stents.[25] With regard to the extent of neointimal hyperplasia, a resembling result was showed in the BIOFLOW- $₫$ trial although the trial was not conducted only in ACS patients and the extent neointimal hyperplasia was estimated with the neointimal area not by the OCT but intravascular ultrasound.[26]

In this study, the mean neointimal thickness of the O-SESs had a tendency to be thinner than X-EESs, but the difference did not reach a statistical significance at the 8-month follow-up. In the BIOSTEMI trial, there was no significant difference in clinical indicated TLR at 1year, but a significant difference in the TLR was permitted at the 2 year follow-up. $[4,5]$ TLR was mainly caused by in-stent restenosis mostly due to neointimal hyperplasia.[27] Therefore, the difference in the clinical outcome between the 1 year follow-up and 2 year follow-up might be driven by the degree of neointimal hyperplasia. Moreover, the mechanism in which a thinner neointimal thickness was formed in the O-SESs than X-EES may be explained by the resolution of the polymer. We evaluated only the 8-month follow-up OCT findings, but, with a long-term follow up, the statistical significance may appear with respect to the mean neointimal thickness.

An advantage of the O-SESs shown by the results of the present study was observed at the 8-month follow up, in which the duration that the poly-L-lactic acid polymer used in the O-SESs had not been degraded, because the polymer of the 0-SESs generally extends well beyond a year.[8] Therefore, ultrathin struts may play a key role in a better vascular healing and thin neointimal thickness. In addition, since the polymer used in the O-SESs degrades between one and two years after stenting, the vascular condition may be more advanced as compared to X-EES, which may partially explain the favorable 2-year outcomes of the BIOSTEMI trial.[5]

\section{Clinical implication}

Compared to X-EESs, O-SESs had a better mid-term vascular healing and tended to have a thinner neointima in ACS patients. The use of O-SESs may further improve late stent failure such as stent thrombosis and in stent restenosis in acute ACS patients undergoing primary $\mathrm{PCl}$.

\section{Limitations}

Our study had several limitations. First, this study was a retrospective, non-randomized, single center and small population sized study. Second, the OCT follow up was performed at 8 months, in which the polymer did not degrade during that period. Therefore, a long-term OCT follow up of the O-SESs as 
compared to the X-EESs is needed to elucidate the effect of the biodegradable polymer and siliconcarbide passive layer.

\section{Conclusions}

Compared to X-EESs, O-SESs had a better mid-term vascular healing and tended to have a thinner neointima in ACS patients, which may be one of the possible mechanisms of the lower TLR in O-SESs than X-EESs.

\section{Declarations}

\section{Acknowledgements}

The authors thank Mr. John Martin for his linguistic assistance with this manuscript.

\section{Funding}

We have no funding.

\section{Compliance with ethical standards}

\section{Conflict of interest statement}

We have no conflict of interest.

\section{Research involving human participants and/or animals}

All procedures performed in this study involving human participants were in accordance with the ethical standards of the institutional research committee and the 1964 Helsinki Declaration and its later amendments or comparable ethical standards.

\section{Informed consent}

Written informed consent was obtained from all participating patients and the protocol was approved by the local ethics committee.

\section{References}

1. Ibanez B, James S, Agewall S, Antunes MJ et al (2018) 2017 ESC Guidelines for the management of acute myocardial infarction in patients presenting with ST-segment elevation: The Task Force for the management of acute myocardial infarction in patients presenting with ST-segment elevation of the European Society of Cardiology (ESC). European heart journal 39(2):119-177

2. Kastrati A, Dibra A, Spaulding C et al (2007) Meta-analysis of randomized trials on drug-eluting stents vs. bare-metal stents in patients with acute myocardial infarction. European heart journal 
28(22):2706-2713

3. de Waha A, King LA, Stefanini GG et al (2015) Long-term outcomes of biodegradable versus durable polymer drug-eluting stents in patients with acute ST-segment elevation myocardial infarction: a pooled analysis of individual patient data from three randomised trials. Eurolntervention: journal of EuroPCR in collaboration with the Working Group on Interventional Cardiology of the European Society of. Cardiology 10(12):1425-1431

4. Iglesias JF, Muller O, Heg D et al (2019) Biodegradable polymer sirolimus-eluting stents versus durable polymer everolimus-eluting stents in patients with ST-segment elevation myocardial infarction (BIOSTEMI): a single-blind, prospective, randomised superiority trial. Lancet 394(10205):1243-1253

5. Pilgrim T, Muller O, Heg D et al (2021) Biodegradable- Versus Durable-Polymer Drug-Eluting Stents for STEMI: Final 2-Year Outcomes of the BIOSTEMI Trial. JACC Cardiovascular interventions 14(6):639648

6. Bezerra HG, Attizzani GF, Sirbu V et al (2013) Optical coherence tomography versus intravascular ultrasound to evaluate coronary artery disease and percutaneous coronary intervention. JACC Cardiovascular interventions 6(3):228-236

7. Nakamura D, Attizzani GF, Toma C et al (2016) Failure Mechanisms and Neoatherosclerosis Patterns in Very Late Drug-Eluting and Bare-Metal Stent Thrombosis. Circulation Cardiovascular interventions $9(9)$

8. Iglesias JF, Roffi M, Degrauwe S et al (2017) Orsiro cobalt-chromium sirolimus-eluting stent: present and future perspectives. Expert Rev Med Dev 14(10):773-788

9. Nakamura D, Yasumura K, Nakamura H et al (2019) Different Neoatherosclerosis Patterns in DrugEluting- and Bare-Metal Stent Restenosisãه- Optical Coherence Tomography Study. Circulation journal: official journal of the Japanese Circulation Society 83(2):313-319

10. Matsuhiro Y, Matsunaga-Lee Y, Nakamura D et al (2019) Characteristics of abnormal post-stent optical coherence tomography findings in hemodialysis patients. Catheterization cardiovascular interventions: official journal of the Society for Cardiac Angiography Interventions 94(7):956-963

11. Guagliumi G, Shimamura K, Sirbu V et al (2018) Temporal course of vascular healing and neoatherosclerosis after implantation of durable- or biodegradable-polymer drug-eluting stents. European heart journal 39(26):2448-2456

12. Niccoli G, Montone RA, Di Vito L et al (2015) Plaque rupture and intact fibrous cap assessed by optical coherence tomography portend different outcomes in patients with acute coronary syndrome. European heart journal 36(22):1377-1384

13. Tearney GJ, Regar E, Akasaka T et al (2012) Consensus standards for acquisition, measurement, and reporting of intravascular optical coherence tomography studies: a report from the International Working Group for Intravascular Optical Coherence Tomography Standardization and Validation. J Am Coll Cardiol 59(12):1058-1072 
14. Soeda T, Uemura S, Park SJ et al (2015) Incidence and Clinical Significance of Poststent Optical Coherence Tomography Findings: One-Year Follow-Up Study From a Multicenter Registry. Circulation 132(11):1020-1029

15. Cassese S, Xhepa E, Ndrepepa G et al (2018) Vascular response to percutaneous coronary intervention with biodegradable-polymer vs. new-generation durable-polymer drug-eluting stents: a meta-analysis of optical coherence tomography imaging trials. European heart journal Cardiovascular Imaging 19(11):1294-1301

16. Nakamura D, Lee Y, Yoshimura T et al (2014) Different serial changes in the neointimal condition of sirolimus-eluting stents and paclitaxel-eluting stents: an optical coherence tomographic study. Eurolntervention: journal of EuroPCR in collaboration with the Working Group on Interventional Cardiology of the European Society of Cardiology 10(8):924-933

17. Matsuhiro Y, Nakamura D, Shutta R et al (2021) Difference of vascular healing between bioabsorbable-polymer and durable-polymer new generation drug-eluting stents: an optical coherence tomographic analysis. Int J Cardiovasc Imaging 37(4):1131-1141. doi:10.1007/s10554020-02094-y

18. Morice MC, Serruys PW, Sousa JE et al (2002) A randomized comparison of a sirolimus-eluting stent with a standard stent for coronary revascularization. N Engl J Med 346(23):1773-1780

19. Song HG, Kang SJ, Ahn JM et al (2014) Intravascular ultrasound assessment of optimal stent area to prevent in-stent restenosis after zotarolimus-, everolimus-, and sirolimus-eluting stent implantation. Catheterization cardiovascular interventions: official journal of the Society for Cardiac Angiography Interventions 83(6):873-878

20. Hong MK, Mintz GS, Lee CW et al (2006) Intravascular ultrasound predictors of angiographic restenosis after sirolimus-eluting stent implantation. European heart journal 27(11):1305-1310

21. Kolandaivelu K, Swaminathan R, Gibson WJ et al (2011) Stent thrombogenicity early in high-risk interventional settings is driven by stent design and deployment and protected by polymer-drug coatings. Circulation 123(13):1400-1409

22. Kastrati A, Mehilli J, Dirschinger $J$ et al (2001) Intracoronary stenting and angiographic results: strut thickness effect on restenosis outcome (ISAR-STEREO) trial. Circulation 103(23):2816-2821

23. Kobayashi S, Inoue N, Ohashi Y et al (2003) Interaction of oxidative stress and inflammatory response in coronary plaque instability: important role of C-reactive protein. Arteriosclerosis, thrombosis, and vascular biology 23 (8):1398-1404

24. Kandzari DE, Koolen JJ, Doros G et al (2020) Ultrathin Bioresorbable-Polymer Sirolimus-Eluting Stents Versus Thin Durable-Polymer Everolimus-Eluting Stents for Coronary Revascularization: 3Year Outcomes From the Randomized BIOFLOW V Trial. JACC Cardiovascular interventions 13(11):1343-1353

25. Karjalainen PP, Varho V, Nammas W et al (2015) Early neointimal coverage and vasodilator response following biodegradable polymer sirolimus-eluting vs. durable polymer zotarolimus-eluting stents in 
patients with acute coronary syndrome -HATTRICK-OCT trial. Circulation journal: official journal of the Japanese Circulation Society 79(2):360-367

26. Windecker S, Haude M, Neumann FJ et al (2015) Comparison of a novel biodegradable polymer sirolimus-eluting stent with a durable polymer everolimus-eluting stent: results of the randomized BIOFLOW-II trial. Circulation Cardiovascular interventions 8(2):e001441

27. Byrne RA, Joner M, Kastrati A (2015) Stent thrombosis and restenosis: what have we learned and where are we going? The Andreas Grüntzig Lecture ESC 2014. European heart journal 36(47):33203331

\section{Figures}

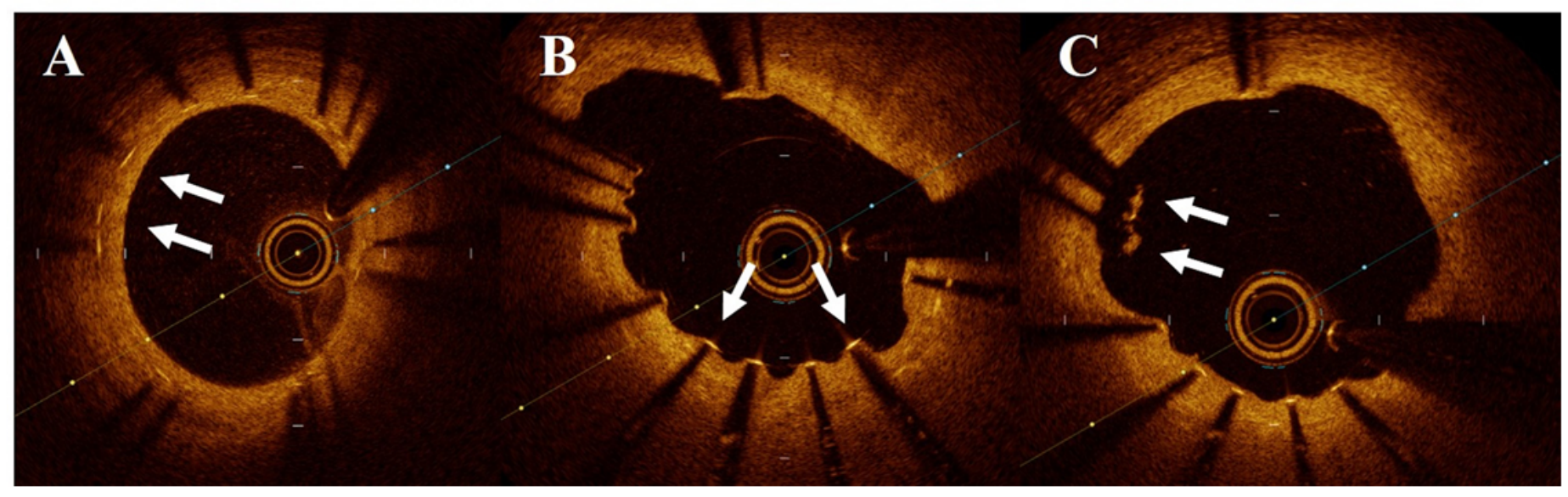

\section{Figure 1}

The representative images of covered, uncovered and malapposed struts. a Covered struts (white arrows), b uncovered struts (white arrows), c malapposed struts (white arrows) 